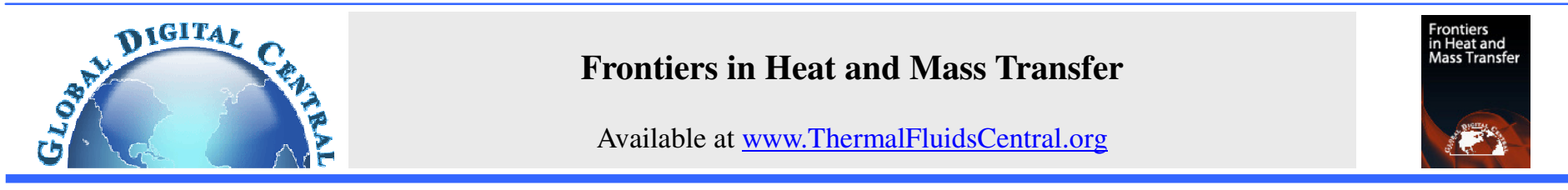

\title{
MICROCANTILEVERS IN BIOMEDICAL AND THERMO/FLUID APPLICATIONS
}

\author{
Khalil Khanafer $^{\mathrm{a}}$, Kambiz Vafai ${ }^{\mathrm{b}, *}$ \\ ${ }^{a}$ Vascular Mechanics Lab., Biomedical Engineering Department and Section of Vascular Surgery, Ann Arbor, MI 48109, USA \\ ${ }^{b}$ Mechanical Engineering Department, University of California, Riverside, CA 92505, USA
}

\begin{abstract}
A study was conducted to demonstrate the applications of microcantilevers in biomedical and thermo/fluid fields. The deflection of the microcantilevers due to biomaterial and turbulence effects was highlighted in this work. The novel patented microcantilever assemblies that were presented in this study can increase the signal and decrease the unfavorable deflection due to flow disturbances. This work paves the road for researchers in the area microcantilever based biosensors to design efficient microsensor systems that exhibit minimal errors in the measurements. Fluid-structure interaction was also utilized to investigate some aspects of the fluid flow and heat transfer characteristics.
\end{abstract}

Keywords: Fluidic cell; Heat Transfer; Microcantilever; Fluid-Structure Interaction

\section{INTRODUCTION}

Microcantilever sensor systems have been receiving significant attention in various areas of interest ranging from biomedical to thermal applications (Zhang et al., 2003; Ji et al., 2001; Dareinga et al., 2006; Yang et al., 2003; Thundat et al., 1994; Thundat et al., 1995; Lai et al., 1997; Chen et al., 1995). Microsensors containing microcantilevers are shown to be sensitive and accurate. Changes in the physical properties of a microcantilever are used to detect changes in the environment surrounding it. Most often the deflection of the microcantilever is measured to indicate the presence or absence of a certain analyte. Microcantilevers are commonly made of silicon, silicon nitride, metal or combinations thereof. For use in assays for biological or chemical agents, the microcantilevers are commonly a bimaterial, such as gold on one side and silicon on the other side. The gold side is then coated with a receptor that specifically binds to a given analyte (species being measured in an analytical procedure). Receptor/analyte pairs include antibodies and antigens, complementary nucleotide sequences and receptors and small molecules. When the analyte molecules bind to the receptor, the side coated with the receptor will either become tensioned or relieved, thereby causing the microcantilever to deflect. The concentration of the analyte can be determined by the degree of deflection. The amount of deflection is usually in nanometers. This deflection is usually measured using optical techniques.

In biological applications, microcantilever-based biosensors have been used in monitoring hazardous biological and chemical agents, and in screening patients for the presence of diseases and to determine its susceptibility to a given drug (Ji et al., 2001). This is due to their fast responses, high sensitivity, and their potential for an inexpensive arraybased sensing platform (Dareinga et al., 2006). As such, accurately designed biosensors can provide fast and accurate detection of pathogens within a short period of time. A collection of miniaturized biosensors can be arranged on a solid substrate to perform many tests instantaneously so higher throughput and speed can be achieved. This collection of micro-arrays arrangement is often called a biochip. First applications of microcantilever arrays as tools for bimolecular detection have been illustrated in the field of DNA hybridization detection (Thundat et al., 19945; Mosbach and Danielsson, 1974).

In thermal applications, microcantilevers with integrated heaters have been used in various applications such as thermomechanical data storage (Lee and King, 2008; Berger et al., 1998), high density data storage (King et al., 2002; Vettiger et al., 2002; Binnig et al., 1999; King et al., 2001), thermomechanical cantilever actuation (Lee and King, 2007), nanometer-scale manufacturing (Sheehan et al., 2004; Nelson et al., 2006), nanometer-scale thermal measurements (Gotsmann and Duerig, 2005; Gotsmann and Duerig, 2004; Park et al., 2008), and vapor detection (Pinnaduwage et al., 2003).

\subsection{Thermo/Fluid Applications}

Majority of the studies on heat flow from the cantilever to the environment was limited to heat flow along the microcantilever and into the substrate while ignoring heat flow to the air environment (Duerig, 2005; Masters et al., 2005; King, 2005). However, several applications utilized heated microcantilevers that are suspended in air environment and away from a substrate (Lee and King, 2008; Berger et al., 1998; Lee and King, 2007; Sheehan et al., 2004; Nelson et al., 2006; Pinnaduwage et al., 2003). Recently, Kim and King (2009) investigated transient heat conduction between a heated microcantilever and its air environment. Time-averaged heat flow from the cantilever leg to the air was determined to be two to six folds greater than timeaveraged heat flow from the cantilever heater to the surrounding air.

Khanafer and Vafai (2005) investigated numerically the effect of flow conditions and the geometric variation of the microcantilever's supporting system on the microcantilever detection capabilities within a

\footnotetext{
*Corresponding author.Email: Vafai@engr.ucr.edu
} 
fluidic cell for various relevant parameters assuming rigid microcantilever. Their results illustrated that the flow direction had a profound effect on the normal velocity across the microcantilever due to the presence of the supporting mechanism. As such the unfavorable deflection caused by high normal velocity values was expected to be reduced for the flow from the leading edge of the microcantilever (Figs. 1 and 2). In addition, Fig. 2 shows that as Reynolds number increases, the normal velocity decreases for the case where the flow is from left to right towards the tip of the microcantilever.

Khanafer et al. (2004) established the minimum spacing distance between an array of the microcantilevers that produce similar flow conditions around each resulting in an optimum utilization of the biosensor. This minimum spacing distance was essential for the microcantilevers to function independent of each other when they are utilized to detect concentrations of different species to be measured (Fig. 3).

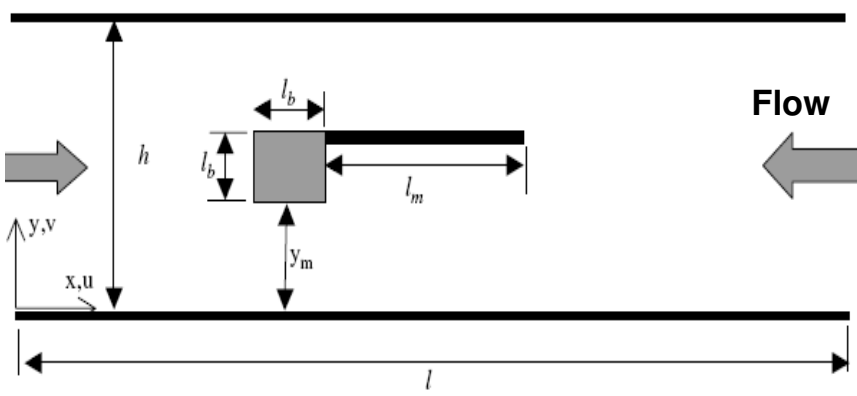

Fig. 1 Physical model and the coordinate system (Khanafer and Vafai, 2005)

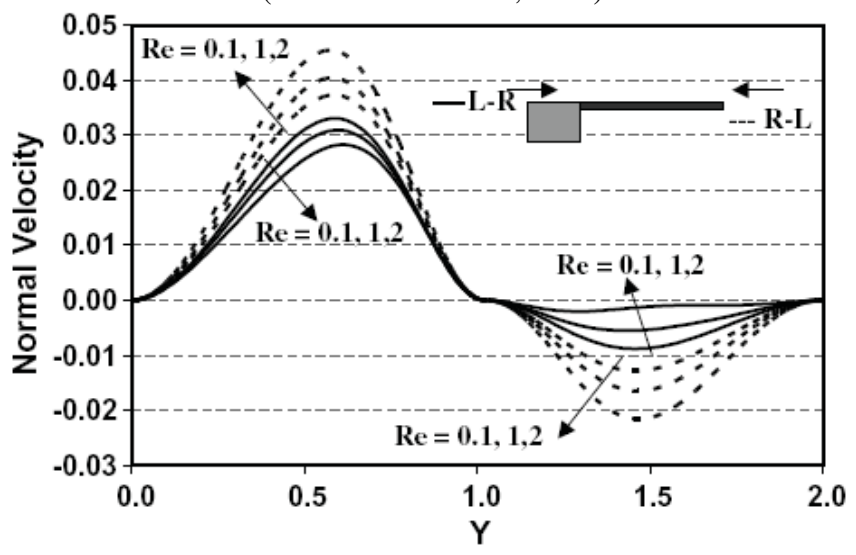

Fig. 2 Effect of the Reynolds number on the normal velocity component along the mid-section of the microcantilever for different flow directions $\left(\mathrm{H}=2, \mathrm{~L}_{\mathrm{b}}=0.05\right)$ (Khanafer and Vafai, 2005)

Figure 3 illustrates the optimum distance between multiple microcantilevers, as established in their investigation, in order to obtain identical flow conditions. Khaled and Vafai (2004) studied numerically the effect of microcantilever inclination on analyte adhesion. A generalized model for the analyte adhesion was considered based on wall shear stress (WSS) at the microcantilever surface. Several analytical solutions for special cases were obtained. They found that the total mass transfer was enhanced by increasing the adhesion rate, the Peclet number and the use of converging flows over the microcantilever (Fig. 4).

Further, it was found that there exists a critical Peclet number that can maximize the total mass transfer when WSS slowed down the adhesion process (Fig. 5). Correlations were established on the basis of the numerical simulations for predicting flow operating conditions inside fluidic cells under maximized mass transfer rate conditions.
The phenomenon of bimetallic effect is referred to a condition where the silicon microcantilevers with a thin gold film on one side undergo measurable bending in response to temperature changes. In that particular case, the differential stress in the microcantilever is created due to dissimilar thermal expansion coefficients of the silicon substrate and the gold coating.

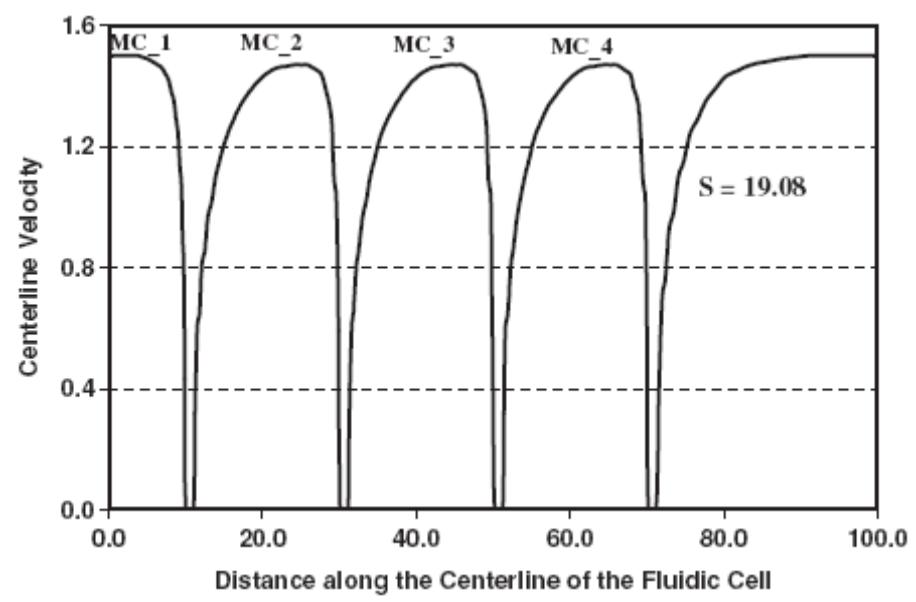

Fig. 3 Effect of Effect of the spacing distance between an array of four microcantilevers on the centerline velocity $\mathrm{U}(\mathrm{X}, \mathrm{H} / 2): H=10$, $R e=2.5, H a=0, Y_{m}=5$ (Khanafer et al., 2004)
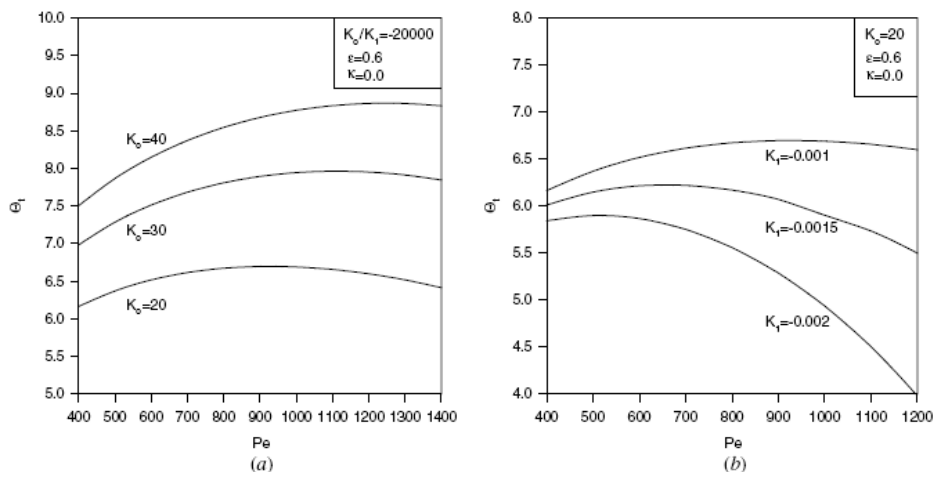

Fig. 4 Effect of Variations of the dimensionless total mass transferred $\Theta_{\mathrm{t}}$ with $(a) K_{\mathrm{o}}$ and the Peclet number $P_{e}$ at a fixed value of $K_{1} / K_{\mathrm{o}}$ and $(b) P_{e}$ and $K 1$ (Khaled and Vafai, 2004).

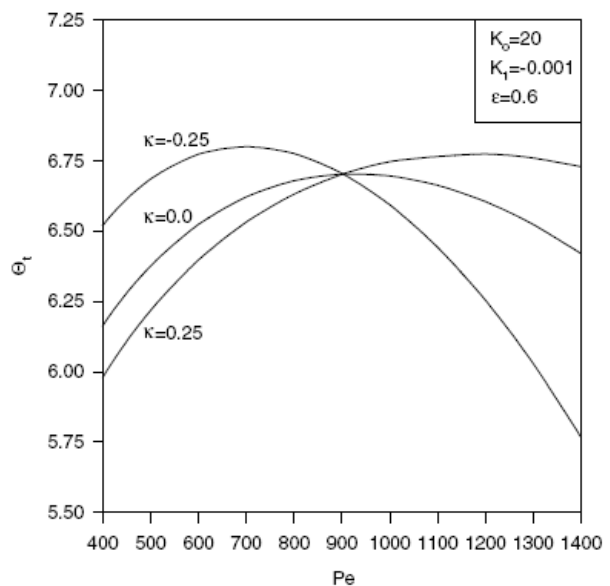

Fig. 5 Variations of the dimensionless total mass transferred $\Theta_{t}$ with the Peclet number $P e$ and the dimensionless slope $\kappa$. (Khaled and Vafai, 2004) 
A theoretical model for predicting the deflection and force of a bimaterial cantilever was presented by Ramos et al. (2007). Their approach predicted a non linear thermal dependence of cantilever strain. This theoretical conclusion was experimentally validated. Rinaldi et al. (2007) proposed a method to test MEMS cantilevers under variant electro-thermal influences in order to quantify the effective boundary support condition obtained for a foundry process. A non-contact optical sensing approach was employed for the dynamic testing. The Rayleigh-Ritz energy method using boundary characteristic orthogonal polynomials was employed for the modeling and theoretical analysis.

Khaled et al. (2003) investigated the main causes for the deflection of microcantilevers embedded in micromechanical biodetection systems. Their results showed that the oscillating flow conditions, which were the main source of turbulence produced substantial deflections at relatively large frequencies. Bimaterial effects influencing the microcantilever deflections were established analytically, and found to be prominent at a relatively low frequency of turbulence (Fig. 6). In the absence of bimaterial effects, turbulence increased the deflection due to chemical reactions at relatively large frequency of turbulence yet it increased the noise due to the increased dynamical effects of the flow on the microcantilever. Novel microcantilever assemblies were presented for the first time that can increase the deflection due to chemical reaction while decreasing those due to flow dynamical effects as depicted in Fig. 7. Two major patents were established (Vafai and Khaled, 2010; Vafai et al., 2007).

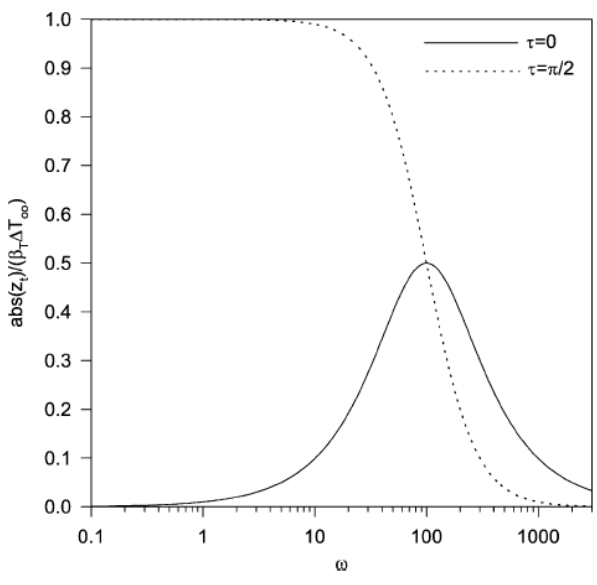

Fig. 6 Effects of $\omega$ on $\mathrm{l}_{\mathrm{t}} \mathrm{l} /\left(\beta_{\mathrm{T}} \Delta \mathrm{T}_{\infty}\right)($ Khaled et al., 2003).

Jana et al. (2007) developed a semianalytical theoretical model for estimating viscous flow-induced deformations of microcantilevers. Their model was useful in understanding the hydrodynamic drag on a microcantilever moving through a fluid at a constant speed, an important consideration in single molecule force spectroscopy. Wu et al. (2007) studied numerically the effect of flow velocity on microcantilever-based biosensor using both rectangular and triangular shapes. It was found numerically that the transportation of analyte, reactive rate, the distribution of concentration and deflection in the $\mathrm{z}$ axis were all effected by changing the flow velocity. Their results had shown that flow velocity was an important factor for this biosensor. An analytical approach to determine the streamlines of fluid flow adjacent to the surfaces of vibrating cantilevers was presented by Dareing et al. (2006).

Fluid flow over the top and bottom surfaces of a microcantilever was established by solving two-dimensional Navier-Stokes equations for viscous flow. The $\mathrm{x}$ and $\mathrm{y}$ velocity components were used to establish streamlines for absolute fluid motion. These streamlines showed a central stagnation core perpendicular and central to the cantilever surface extending along the full length of cantilevers, which most likely accounts for the added mass effect (induced mass) of fluid media around vibrating microcantilevers. The dynamic response of a microcantilever beam used as a transducer in a biomechanical sensor for molecule detection was analyzed by Decuzzi et al. (2007). The cantilever motion was modeled using the classical Euler-Bernoulli beam theory coupled to the Reynolds equation of lubrication accounting for the hydrodynamic interactions. The beam was oscillated by an electrostatic harmonic force in close proximity to a rigid wall in an analysis chamber. It is shown that the dynamic response of the beam can be accurately predicted by a simple equivalent damped harmonic oscillator whose quality factor has been estimated as a function of the beam-wall gap. As the gap is reduced the quality factor is reduced and damping becomes more and more important. A transduction relationship between the frequency shift and the mass of molecules adherent on the beam is proposed which accounts for the beam-fluidsubstrate interactions.

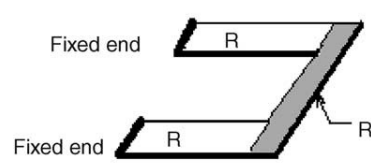

(a)

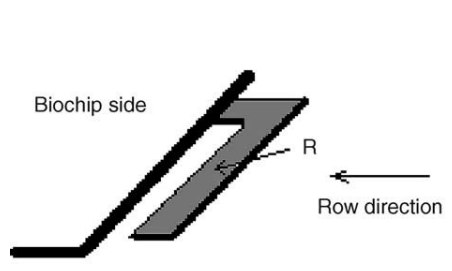

(c) (b)

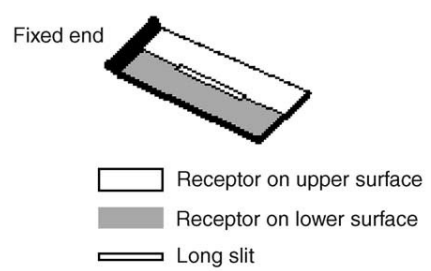

(d)

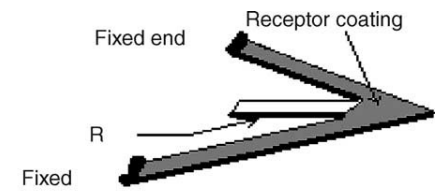

Fig. 7 Suggested different designs of the microcantilever assembly (Vafai and Khaled, 2010; Vafai et al., 2007, Khaled et al., 2003)

Efforts to improve the sensitivity and throughput of microcantilever sensors in flowing fluids were primarily aimed in the literature toward fabricating more flexible microcantilevers and enabling higher flow rates. Vafai and co-workers (Khaled et al., 2003; Vafai and Khaled, 2010; Vafai et al., 2007) suggested new generations of microcantilevers that are less sensitive to turbulence and have enhanced deflections. Assembly (a) and (b) of Fig. 7 was found to have a large effective stiffness hence lower turbulence effects. Also, they have larger deflection as compared to an ordinary microcantilever. Specifically, there is a substantial enhancement in deflection at the mid point of the connecting beam for assembly (a) and at the free end of the intermediate beam and for assembly (b). The receptor coatings on the connecting and intermediate beams for assemblies (a) and (b), respectively, are on the opposite surface to those for the other beams where their receptor coatings are on one side as shown in Fig. 7. Assembly (c) will be subjected to lower drag amplitude than the ordinary microcantilever because it is close to the wall. Assembly (d) is an ordinary microcantilever with the receptor coating being placed on one half of the upper surface of the microcantilever and along the opposite half of the lower surface of the microcantilever. This slit allows the separated sides of the microcantilever to have deflections in opposite directions upon analyte bindings with the receptors on the shown alternating surfaces.

Table 1 shows a comparison between the suggested microcantilever assemblies and the ordinary microcantilever, MC, according to their corresponding values of $z_{\mathrm{s}}$ (deflection due to surface stress), $z_{\mathrm{t}}$ (deflection due to bimaterial) and $z_{\mathrm{d}}$ (deflection due to dynamical effects). Note that the length of the intermediate beam for assembly (b) is assumed to extend to the fixed end. 
Table 1 Summary of the performance of the different microcantilever assemblies (Khaled et al., 2003)

\begin{tabular}{|c|c|c|c|c|c|}
\hline $\mathrm{z}$ & Ordinary MC & $\begin{array}{l}\text { Assembly } \\
\text { (a) }\end{array}$ & $\begin{array}{l}\text { Assembly } \\
\text { (b) }\end{array}$ & Assembly (c) & $\begin{array}{l}\text { Assembly } \\
\text { (d) }\end{array}$ \\
\hline Maximum $z_{s}$ & $\begin{array}{c}\mathrm{Z}_{\mathrm{o}} \\
\mathrm{MC} \text { length }=\mathrm{L}\end{array}$ & $\begin{array}{c}1.25 \mathrm{z}_{\mathrm{o}} \\
\text { beam length }=\mathrm{L}\end{array}$ & $\begin{array}{c}\approx 2 \mathrm{z}_{\mathrm{o}} \\
\text { intermediate } \\
\text { beam length }=\mathrm{L}\end{array}$ & $\begin{array}{c}\mathrm{z}_{\mathrm{o}} \\
\text { total length }=\mathrm{L}\end{array}$ & $\begin{array}{c}\Delta \mathrm{z}_{\mathrm{o}}<\mathrm{z}_{\mathrm{o}} \\
\text { slit length }=\mathrm{L}\end{array}$ \\
\hline $\mathrm{z}_{\mathrm{t}}$ & $z_{\text {to }}$ & $1.25 \mathrm{z}_{\mathrm{to}}$ & $\approx 2 \mathrm{z}_{\text {to }}$ & $1.0 z_{\text {to }}$ & $\Delta \mathrm{z}_{\mathrm{t}}<\mathrm{z}_{\text {to }}$ \\
\hline $\mathrm{z}_{\mathrm{d}}$ & $\mathrm{z}_{\mathrm{do}}$ & $<\mathrm{z}_{\mathrm{do}}$ at low $\omega$ & $<\mathrm{Z}_{\mathrm{do}}$ at low $\omega$ & $<\mathrm{z}_{\mathrm{do}}$ & $\approx 0$ \\
\hline
\end{tabular}

Previous studies in the literature assumed rigid microcatilevers when analyzing the flow characteristics in a fluidic cell (Khaled and Vafai, 2004; Khaled and Vafai, 2007; Mahjoob et al., 2008; Vafai and Khaled, 2010a; Vafai and Khaled, 2010b). However, since these devices are extremely small they deflect as a result of flow turbulence and the bimetallic effect in addition to the microcantilever's deflection caused by the binding of the target molecule with the receptor and thus interferes with the target molecule detection process. Due to the high cost and time involved in fabricating microcantilevers for experimental analysis, and with the advancement in computational fluid dynamics, numerical techniques are considered essential in studying the performance of a Biochip. Another advantage of numerical methods is the ability to evaluate numerous variables simultaneously whereas experimental procedures usually involve testing of one variable per model.

Recently, Khanafer et al. (2010) analyzed the effects of the flow conditions and the geometric variation of the microcantilever's bluff body on the microcantilever detection capabilities within a fluidic using a finite element fluid-structure interaction (FSI) model. Their results showed that low inlet fluid velocity condition exhibited no vortices around the microcantilever. However, the introduction of a random noise in the fluidic cell was found to cause the microcantilever to oscillate in a harmonic mode at low velocity. The results of their study showed that microcantilevers excited earlier for large height compared with smaller heights of the bluff body at high inlet fluid velocity (Fig.8).

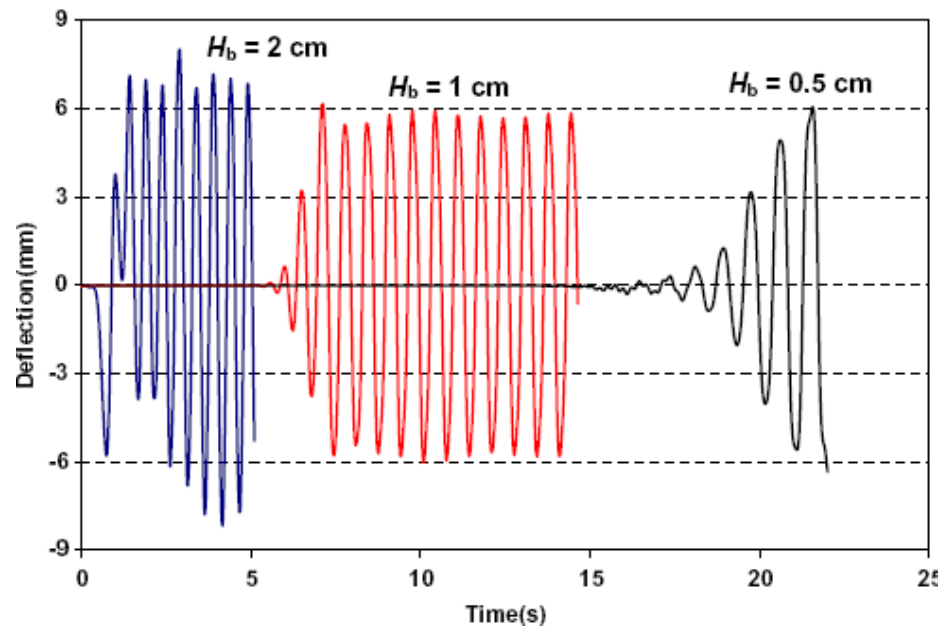

Fig. 8 Effect of the bluff body's height on the deflection of the microcantilever's tip $\left(\mathrm{U}_{\mathrm{o}}=50 \mathrm{~cm} / \mathrm{s}\right)($ Khanafer et al., 2010).

The effect of the inlet velocity magnitude on the streamlines and isotherms is demonstrated in Fig. 9. At low velocity, Fig. 9 shows the absence of vortices around the microcantilever. In addition, the flow is symmetric around the microcantilever when considering low velocity values. As the fluid flows past the bluff body at high velocity $\left(U_{0}=50 \mathrm{~cm} / \mathrm{s}\right)$, vortices develop and shed periodically, thereby inducing vibration in the flexible structure and causing it to oscillate in a harmonic fashion as shown in Fig. 9. This figure manifests that high velocity flow may cause significant unfavorable deflection when placing microcantilevers under high-flow rate sensing conditions. The effect of varying the inlet fluid velocity on the temperature distribution around the microcantilever is depicted in Fig. 9. This figure demonstrates that the temperature is almost uniform everywhere in the channel at high velocity except in a region close to the microcantilever. This is due to a fact that convective heat transfer is dominant compared with conduction mode at high velocity. The region close to the tip of the microcantilever is significant since any unfavorable temperature distribution due to the effect of flow turbulence may affect the design and operation of microcantilever heater.
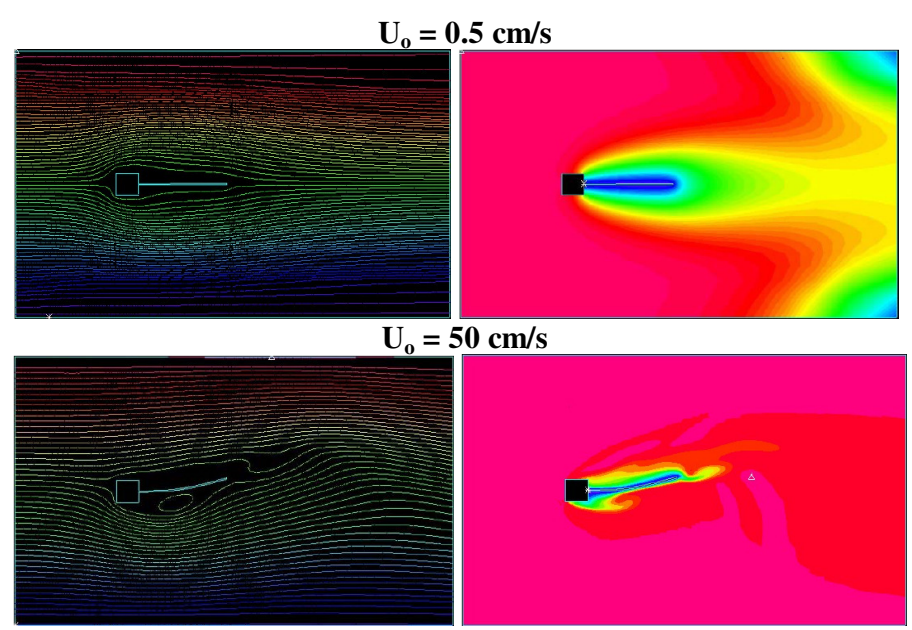

Fig. 9 Effect of the flow velocity magnitude on the streamlines and isotherms around the microcantilever (Khanafer et al., 2010).

\subsection{Biomedical Application}

Biosensors are characterized by rapid responses and high sensitivity (Baller et al, 2000; Thundat et al., 1994). As such, biosensors properly designed can provide fast and accurate detection of pathogens within a short period of time. A collection of miniaturized biosensors can be arranged on a solid substrate to perform many tests at the same time so higher throughput and speed can be achieved. This collection of microarrays is often called a biochip. First applications of microcantilever arrays as tools for biomolecule detection have been illustrated in the field of DNA hybridization detection (Thundat et al., 1995; Mosbach and Danielsson, 1974). The superior capabilities of the microcantilevers to detect a specific substance below the detection limits of classical methods have been studied in the literature. Fritz et al. (2000) conducted a study on the transduction of DNA hybridization and receptor-ligand binding into a direct nanomechanical response of microfabricated cantilevers. The deflection of the microcantilevers was found to provide a true molecular recognition signal. Baller et al. (2000) presented quantitative and qualitative detection of analyte vapors using a microcantilever array to observe transduction of physical and chemical processes into nano-mechanical motion of the microcantilever. The measurements of surface stress change due to protein adsorption on a cantilever array were shown by Baller et al. (2000). Hansen et al. (2001) demonstrated the discrimination of DNA mismatches using simple microcantilever-based optical deflection assay. Wu et al. (2001) used different geometries of microcantilevers to detect two forms of prostate-specific antigen (PSA) over a wide range of concentrations. Hagan et al. (2002) examined microcantilever deflections resulting from adsorption and subsequent hybridization of DNA molecules using an empirical potential. The authors in their study found that the dominant contribution to these deflections arises from hydration forces and not conformational entropy or electrostatics. Label-free protein assay based on microcantilevers array was demonstrated by Arntz et al. (2003). This method allowed biomarker proteins to be detected via measurements of surface stress generated by 
antigen-antibody molecular recognition. Glucose biosensing using an enzyme-coated microcantilever was studied by Subramanian et al. (2002). The enzyme glucose oxide was immobilized on a micromachined silicon cantilever containing a gold coating. Quantifiable deflection of the microcantilevers was observed in the presence of an appropriate analyte. Analysis of the reaction energetics and the expected thermal response of the microcantilever indicated that the deflection was not a result of reaction-generated heat but resulted from surface induced stresses (Khaled et al., 2003; Vafai and Khaled, 2010; Vafai et al., 2007).

\section{FLUID STRUCTURE INTERACTION}

Significant advances have been made during the last years in the development and use of computational methods for fluid flows with structural interactions (Khanafer et al., 2010; Bathe and Ledezma, 2007; Bathe and Zhang, 2002; Kohno and Bathe, 2005ab; Kohno and Bathe, 2006; Khanafer and Berguer, 2009; Khanafer et al., 2009; Zhang et al., 2003; Bathe and Zhang, 1999; Bathe and Zhang, 2004). These advances pertain to the continuous efforts to reach more efficient computational techniques in solving complex problems ( $\mathrm{Lim}$ and $\mathrm{Li}$, 2007; Kambouchev et al., 2007; Tijsseling, 2007; Baylot and Bevins, 2007; Dailey et al., 2007). Fluid-structure interaction plays an important role in many different types of real-world situations and industrial applications such as biomedical, material processing, automotive, aeronautical and civil engineering. In an FSI application, the stresses and deformations of a given structure are computed simultaneously with the flow and heat-transfer variables of a fluid that surrounds the structure. Such stresses and deformations are occasionally due to temperature gradients and/or temperaturedependent structural properties. In FSI analyses, fluid forces are acting onto the solid and the solid deformation changes the fluid domain. In most interaction scenarios, the computational domain is divided into the fluid domain and solid domain, where a fluid model and a solid model are defined through their material data, boundary conditions, etc (Bathe, 2003; Bathe, 2005; Bathe and Zhang, 2004).

\subsection{Problem Complexity and Displacement Size}

FSI scenarios can be either simple or complex. For FSI problems that involve small displacements, the fluid-flow problem can be solved assuming that the structural displacement does not affect the flow field. That is, the displacements and stresses of the structural configuration are affected by the flow field, but the flow field is not affected by the structure. This is always the case for the microcantilevers. In fact, the effect of solid on the fluid part is negligible. Furthermore, the effect of the fluid on the solid is quite limited when compared to a piezoelectric vibrations of the microcantilever. Then only the fluid stress needs to be applied onto the structure and no iteration between the fluid and solid models is needed. We call this type of interaction "one-way coupling".

As a general rule, displacements are considered large only if they are of the same order of magnitude of the smallest length scale that is important in the simulation. Therefore, for FSI problems that involve large displacements, the flow field is affected by structural deformations and the problem is considered complex. As a result, the fluid and structure exhibit a two-way coupling, and it is necessary to re-solve the fluid-flow problem after each update of the structural configuration. In this sense, the FSI problem can be considered as a moving-boundary problem. An additional level of nonlinearitiy is introduced in such problems, because the fluid and structural problem must be solved iteratively.

Complex problems include those that involve large structural deformations, changes in topology, contact problems, and geometric and/or material nonlinearities. The complexity of any given FSI problem is determined, in large part, by the extent to which the structural component is displaced. The most appropriate and cost effective way to approach such problems is to set up and solve the fluid-flow and structural problems as two completely separate problem steps.

\section{GOVERNING EQUATIONS OF FLUID FLOWS WITH STRUCTURAL INTERACTIONS}

In this section, we briefly present the mathematical model of the fluidstructure interaction fluid flow problems and the descritization of the finite element method. An arbitrary Lagrangian-Eulerian (ALE) formulation was employed to describe the fluid flow for FSI problems.

\subsection{Equations Governing the Fluid}

Consider an open bounded fluid domain $\Omega^{F}(t)$ with boundary $\Gamma=\Gamma_{D} \cup \Gamma_{N} \cup \Gamma_{I}$ where $\Gamma_{D}$ is the Dirichlet boundary of the fluid, $\Gamma_{N}$ is the Neumann boundary of the fluid, and $\Gamma_{I}$ is the fluid-structure interface boundary. The governing equations for the fluid domain are the continuity and Navier-Stokes equations can be written as

$\rho \frac{\partial u_{i}}{\partial t}+\rho u_{i, j}\left(u_{j}-\hat{u}_{j}\right)=\sigma_{i j, j}+\rho f_{i} \quad$ in $\quad \Omega^{F}(t)$
$u_{j, j}=0$

where $u_{i}$ is the velocity tensor, $\rho$ is the density, $\sigma_{i j}$ is the stress tensor, $f_{i}$ is the body force per unit mass, $\hat{u}$ is the mesh velocity (i.e. moving velocity of the solid region), and the indices indicate vector components and differentiation in index (notation). For a fluid, the stress tensor, $\sigma_{i j}$, can be written as

$\sigma_{i j}=-p \delta_{i j}+\tau_{i j}$

Where $\mathrm{p}$ is the pressure, $\tau_{i j}$ is the deviatoric stress tensor, and $\delta_{i j}$ is the Kronecker delta. For viscous, incompressible fluids, the constitutive relation has the form

$\tau_{i j}=2 \mu s_{i j}$

where $S_{i j}$ is the strain rate tensor defined as

$s_{i j}=\frac{1}{2}\left(u_{i, j}+u_{j, i}\right)$

For incompressible fluids, the principle of conservation of thermal energy is expressed by:

$\rho c_{p}\left(\frac{\partial T}{\partial t}+u_{i} T_{, i}\right)=\left(k T_{, i}\right)_{i}$

where $T$ is the temperature, $k$ is the thermal conductivity of the fluid, and $c_{p}$ is the specific heat at constant pressure.

\subsection{Boundary Conditions}

The boundaries of $\Omega^{F}(t)$ can be assigned any of three types of boundary conditions - velocity, stress, or free-surface (in addition to symmetry). The boundary conditions can be expressed in the following forms:

$$
\begin{aligned}
& u_{i}=\bar{u}_{i} \quad \text { on } \Gamma_{D} \\
& \sigma_{i}=\sigma_{i j} n_{j}=\bar{\sigma}_{i} \quad \text { on } \Gamma_{N} \\
& u_{i}=\bar{u}_{S}^{i} \quad \text { on } \Gamma_{I}
\end{aligned}
$$

where $\bar{u}_{S}^{i}$ are the velocities of the fluid-structure interface $\Gamma_{I}, \bar{u}_{i}$ are the prescribed velocities on $\Gamma_{D}, \bar{\sigma}_{i}$ are the prescribed tractions on $\Gamma_{N}$, $n_{j}$ is the unit outward normal vector to the boundary surface of the fluid. 


\subsection{Equations Governing the Structure}

Elastodynamics Equations

The structural domain of the FSI problem can be described by the following elastodynamics equations:

Momentum Equations

$\rho_{s} \ddot{d}_{i}=\sigma_{s, i j, j}+\rho_{s} \bar{f}_{i} \quad$ in $\quad \Omega^{s}(t)$

$\underline{\text { Equilibrium Conditions }}$

$\sigma_{s, i j} n_{j}=\bar{t}_{S}^{i} \quad$ on $\quad \Gamma_{N}^{S}$

where $\rho_{s}$ is solid density, $\sigma_{s, i j}$ is the solid Cauchy stress tensor, $\bar{f}_{i}$ is the externally applied body force vector at time $\mathrm{t}, \ddot{d}_{i}$ represents the acceleration of the solid region, and $\bar{t}_{S}^{i}$ is the externally applied surface traction vector at time t, $\Omega^{S}(t)$ is the structural domain at time $\mathrm{t}, n_{j}$ is the outward pointing vector on $\Gamma_{N}^{S}$, and $\Gamma_{N}^{S}$ is the boundary of the structural domain at time t.

\section{SOLUTION STRATEGIES}

In its general form, the mathematical modeling of FSI problems requires the simultaneous application of techniques from the fields of computational fluid dynamics, computational structural dynamics, and computational mesh dynamics. It is reported in the literature that the efficient solution of general fluid flow problems at high Reynolds numbers exhibits a major challenge (Gresho and Sani, 2000; Drikakis and Rider, 2005). Bathe and co-workers (Bathe and Pontaza, 2002; Bathe and Zhang, 2002; Kohno and Bathe, 2005) were focused on the development of the flow-condition-based interpolation (FCBI) solution approach, which is a hybrid approach between the usual control volume and finite element methods, drawing on the best features of these techniques. This approach was developed to reach procedures that are stable, accurate and efficient for any Reynolds number, even when rather coarse meshes are used for solution. As discussed Bathe and coworkers (Bathe and Zhang, 2002; Bathe and Zhang, 2004; Kohno and Bathe, 2005; Kohno and Bathe, 2006), the objective in the FCBI formulations was to have good stability and sufficient accuracy for FSI solutions.

\section{CONCLUSIONS}

This review summarizes the applications of microcantilevers in biomedical and thermal/fluid fields. Novel microcantilever assemblies were presented in this review that can increase the deflection due to chemical reaction while decreasing those due to flow dynamical effects. Oscillating flow conditions, which are the main source of turbulence, were found to produce substantial deflections at relatively large frequency of turbulence while bimaterial effects were significant at a relatively low frequency of turbulence. In the absence of bimaterial effects, turbulence increases the deflection due to chemical reactions at relatively large frequency of turbulence yet it increases the noise due to the increased dynamical effects of the flow on the microcantilever. The innovative microcantilever designs that were discussed and are patented are able to substantially increase the signal and decrease the deflection due to flow disturbances. These novel innovative microcantilever's will produce far higher signal to noise ratios. This review also presented the applications of fluid-structure interaction in the design and operation of microcantilever in fluidic cell for monitoring biohazard materials and screening patients for diseases.

\section{NOMENCLATURE}

$b$

$c_{p}$

$\ddot{d}_{i}$

E

$\mathrm{f}_{\mathrm{i}}$

$\overline{\mathrm{f}}_{\mathrm{i}}$

$H_{b}$

$\mathrm{k}$

Pr

$s_{i j}$

$\mathrm{u}_{i}$

$\hat{u}_{\mathrm{j}}$

$T$

$t$

$\mathrm{U}_{\circ}$

$u, v$

W

$x, y$

Greek Symbols

$\rho$

$\rho_{\mathrm{s}}$

$\gamma$

$v$

$\Gamma_{D}$

$\Gamma_{N}$

$\Gamma_{I}$

$\Omega^{F}(t)$

$\sigma_{\mathrm{ij}, \mathrm{j}}$

$\bar{\sigma}_{\mathrm{ij}, \mathrm{j}}$

$\sigma_{\mathrm{f}}$

$\sigma_{\mathrm{s}}$

Subscripts

$c$

H

$f$

$s$ thickness of the microcantilever (m)

specific heat at constant pressure $(\mathrm{J} / \mathrm{kg} \cdot \mathrm{K})$

acceleration of the solid region $\left(\mathrm{m}^{2} / \mathrm{s}\right)$

Young's modulus $\left(\mathrm{N} / \mathrm{m}^{2}\right)$

fluid body force per unit mass

the externally applied body force vector at time $t$

height of the bluff body (m)

thermal conductivity $(\mathrm{W} / \mathrm{m} \cdot \mathrm{K})$

Prandtl number

strain rate tensor

fluid velocity tensor

mesh velocity $(\mathrm{m} / \mathrm{s})$

temperature $(\mathrm{K})$

time (s)

inlet velocity $(\mathrm{m} / \mathrm{s})$

$x$ - and $y$-component velocities $(\mathrm{m} / \mathrm{s})$

length of the microcantilever (m)

$x$ - and $y$-coordinates (m)

fluid density $\left(\mathrm{kg} / \mathrm{m}^{3}\right)$

solid density $\left(\mathrm{kg} / \mathrm{m}^{3}\right)$

iteration

Poison's ratio

Dirichlet boundary of the fluid

Neumann boundary of the fluid

fluid-structure interface boundary

fluid domain

fluid stress tensor $\left(\mathrm{kg} / \mathrm{m} . \mathrm{s}^{2}\right)$

solid Cauchy stress tensor $\left(\mathrm{kg} / \mathrm{m} . \mathrm{s}^{2}\right)$

traction of the fluid along the interface boundary

traction of the solid along the interface boundary

low temperature

high temperature

fluid

solid

\section{REFERENCES}

Arntz, Y., Seelig, J.D., Lang, H.P., Zhang, J., Hunziker, P., Ramseyer, J.P., Meyer, E., Hegner, M., Gerber, C., 2003, "Label Free Protein Assay Based on a Nanomechanical Cantilever Array," Nanotechnology 14, 86-90.

doi:10.1088/0957-4484/14/1/319

Baller, M.K., Lang, H.P., Fritz, J., Gerber, C., Gimzewski, J.K., Drechsler, U., Rothuizen, H., Despont, M., Vettiger, P., Battiston, F.M., Ramseyer, J.P., Fornaro, P., Meyer, E.,. Guntherodt, H.-J., 2000,” A Cantilever Array-Based Artificial Nose," Ultramicroscopy 82, 1-9. doi:10.1016/S0304-3991(99)00123-0

Bathe K.J., Zhang, H., 2002, "A Flow-Condition-Based Interpolation Finite Element Procedure for Incompressible Fluid Flows," Computers and Structures 88, 1267-1277. doi:10.1016/S0045-7949(02)00077-9 
Bathe KJ, editor. Computational Fluid and Solid Mechanics 2003. Elsevier; 2003.

Bathe KJ, editor. Computational Fluid and Solid Mechanics 2005. Elsevier; 2005.

Bathe, K.J, Pontaza, J.P., 2002, “A Flow-Condition-Based Interpolation Mixed Finite Element Procedure for Higher Reynolds Number Fluid Flows," Mathematical Models and Methods in Applied Sciences 12, 525 -539. doi:10.1142/S0218202502001775

Bathe, K.J, Zhang, H, Ji, S., 1999, "Finite Element Analysis of Fluid Flows Fully Coupled with Structural Interactions," Comput Struct 72, $1-16$.

doi:10.1016/S0045-7949(99)00042-5

Bathe, K.J, Zhang, H., 2002, “A Flow-Condition-Based Interpolation Finite Element Procedure for Incompressible Fluid Flows," Computers and Structures 80, 1267-1277. doi:10.1016/S0045-7949(02)00077-9

Bathe, K.J, Zhang, H., 2004, "Finite Element Developments for General Fluid Flows with Structural Interactions," Int J Numer Methods Eng 60, 213-32.

doi:10.1002/nme.959

Bathe, K.J., Ledezma, G.A., 2007, "Benchmark Problems for Incompressible Fluid Flows with Structural Interactions," Computers and Structures 85, 628-644. doi:10.1016/j.compstruc.2007.01.025

Baylot, J.T, Bevins, T.L., 2007, "Effect of Responding and Failing Structural Components on the Air Blast Pressures and Loads on and Inside of the Structure," Comput Struct 85, 891-910. doi:10.1016/j.compstruc.2007.01.001

Berger, R., Kang, H.P., Gerber, C., Gimzweski, J.K., Fabian, J.H., Scandella, L., Meyer, E., Guntherdot, H.J., 1998," Micromechanical Thermogravimetry," Chem. Phys. Lett. 294, 363-369.

doi:10.1016/S0009-2614(98)00817-3

Binnig, G., Despont, M., Drechsler, U., Häberle, W., Lutwyche, M., Vettiger, P., Mamin, H.J., Chui, B.W., Kenny, T.W., 1999," UltrahighDensity Atomic Force Microscopy Data Storage with Erase Capability," Appl. Phys. Lett. 76, 1329-1331. doi:10.1063/1.123540

Chen, G.Y., Thundat, T., Wachter, E.A., Warmack, R.J., 1995," Adsorption-Induced Surface Stress and its Effects on Resonance Frequency of Microcantilevers," J. Appl. Phys. 77, 3618-3622. doi:10.1063/1.359562

Dailey, H.L, Yalcin, H.C, Ghadiali, S.N., 2007, "Fluid-Structure Modeling of Flow-Induced Alveolar Epithelial Cell Deformation," Comput Struct 85,1066-1071.

doi:10.1016/j.compstruc.2006.11.012

Dareing DW, Tian F, Thundat T, 2006," Effective Mass and Flow Patterns of Fluids Surrounding Microcantilevers," Ultramicroscopy 106, 789-794.

doi:10.1016/j.ultramic.2005.11.011

Dareinga, D.W., Tianb, F., Thundatb, T., 2006,“ Effective Mass and Flow Patterns of Fluids Surrounding Microcantilevers, Ultramicroscopy, 106, 789-794.

doi:10.1016/j.ultramic.2005.11.011

Decuzzi, P, Granaldi, A, Pascazio, G, 2007, "Dynamic Response of Microcantilever-Based Sensors in a Fluidic Chamber," Journal of Applied Physics 101, article number 024303.

doi:10.1063/1.2426966

Drikakis, D, Rider, W., 2005, High-Resolution Methods for Incompressible and Low Speed Flows. Springer: Berlin.
Duerig, U., 2005," Fundamentals of Micromechanical Thermoelectric Sensors," J. Appl. Phys. 98, 044906-14. doi:10.1063/1.2006968

Fritz, J., Baller, M.K., Lang, H.P., Rothuizen, H., Vettiger, P., Meyer, E., Guntherodt, H.-J., Gerber, C., Gimzewski, J.K., 2000, ”Translating Biomolecular Recognition into Nanomechanics," Science 288, 316318.

\section{doi:10.1126/science.288.5464.316}

Gotsmann, B., Duerig, U., 2005," Experimental Observation of Attractive and Repulsive Thermal Forces on Microcantilevers, "Appl. Phys. Lett. 87, 194102-3.

doi: $10.1063 / 1.2128040$

Gotsmann, B., Durig, U., 2004,"Thermally Activated Nanowear Modes of a Polymer Surface Induced by a Heated Tip," Langmuir 20, 14951500 .

doi:10.1021/la036112w

Gresho, PM, Sani, RL., 2000, Incompressible Flow and the Finite Element Method. Wiley: New York.

Hagan, M.F., Majumdar, A., Chakraborty, A.K., 2002," Nanomechanical Forces Generated by Surface Grafted DNA," J. Phys. Chem. B 106, 10163-10173.

doi:10.1021/jp020972o

Hansen, K.M., Ji, H.F., Wu, G.H., Datar, R., Cote, R., Majumdar, A., Thundat, T., 2001, "Cantilever-Based Optical Deflection Assay for Discrimination of DNA Single-Nucleotide Mismatches," Anal. Chem. 73, 1567-1571.

doi:10.1021/ac0012748

Jane, A., Raman, A., Dhayal, B., Tripp, S.L., Reifenberger, R.G., 2007, "Microcantilever Mechanics in Flowing Viscous Fluids," Appl. Phys. Letter 90, 114110 -3. doi: $10.1063 / 1.2713238$

Ji, G., Wu, H, Hansen, K., Thundat, T., Datar, R., Cote, R., Hagan, M.F., Chakraborty, A.K., Majumdar, A., 2001, "Origin of Nanomechanical Cantilever Motion Generated from Biomolecular Interactions," Proc. Natl Acad. Sci. USA 98, 1560-1564. doi:10.1073/pnas.031362498

Kambouchev, N, Noels, L, Radovitzky, R., 2007, "Numerical Simulation of the Fluid-Structure Interaction Between Air Blast Waves and Free-Standing Plates," Comput Struct 85, 923-931. doi:10.1016/j.compstruc.2006.11.005

Khaled, A.-R A., Vafai, K., 2004," Optimization Modeling of Analyte Adhesion over an Inclined Microcantilever-Based Biosensors," $J$. Micromech. Microengng. 14, 1220-1229. doi:10.1088/0960$\underline{1317 / 14 / 8 / 015}$

Khaled, A.R.A., and Vafai, K., 2004, "Analysis of Oscillatory Flow Disturbances and Thermal Characteristics Inside Fluidic Cells Due to Fluid Leakage and Wall Slip Conditions" Journal of Biomechanics, 37, 721-729.

doi:10.1016/j.jbiomech.2003.09.017

Khaled, A.R.A., and Vafai, K., 2007, "Analysis of Thermally Expandable Flexible Fluidic Thin Film Channels "ASME Journal of Heat Transfer, 129, 813-818.

doi:10.1115/1.2712853

Khaled, A.-R.A., Vafai, K., Yang, M., Zhang, X., Ozkan, C.S., 2003,” Analysis, Control and Augmentation of Microcantilever Deflections in Bio-Sensing Systems, "Sens. Actuat. B: Chem. 94, 103-115. doi:10.1016/S0925-4005(03)00231-4

Khanafer, K, Alamiri, A, and Pop, I, 2010," Fluid-Structure Interaction Analysis of Flow and Heat Transfer Characteristics Around a Flexible 
Microcantilever in a Fluidic Cell," Int. J. Heat Mass Transfer 53, 16461653.

doi:10.1016/j.ijheatmasstransfer.2010.01.029

Khanafer, K, Berguer, R., 2009, "Fluid-Structure Interaction Analysis of Turbulent Pulsatile Flow within a Layered Aortic Wall as Related to Aortic Dissection," Journal of Biomechanics 42, 2642-2648.

doi:10.1016/j.jbiomech.2009.08.010

Khanafer, K, Bull, JL, Berguer, R, 2009, "Fluid-Structure Interaction of Turbulent Pulsatile Flow Within a Flexible Wall Axisymmetric Aortic Aneurysm Model," European J. Mechanics B-Fluids 28, 88-102. doi:10.1016/j.euromechflu.2007.12.003

Khanafer, K., Khaled, A.-R A., Vafai, K., 2004," Spatial Optimization of an Array of Aligned Microcantilever Biosensors," J. Micromech. Microengng. 14, 1328-1336. doi:10.1088/0960-1317/14/10/006

Khanafer, K., Vafai, K., 2005," Geometrical and Flow Configurations for Enhanced Microcantilever Detection within a Fluidic Cell," Int. J.

Heat Mass Transfer 48, 2886-2895. doi:10.1016/j.ijheatmasstransfer.2004.11.021

Kim, K.J., King, W.P., 2009," Thermal Conduction Between a Heated Microcantilever and a Surrounding Air Environment, Appl. Thermal Engng. 29, 1631-1641.

doi:10.1016/j.applthermaleng.2008.07.019

King, W.P., 2005," Design Analysis of Heated Atomic Force Microscope Cantilevers for Nanotopography Measurements," $J$. Micromech. Microengng. 15, 2441-2448. doi:10.1088/0960-1317/15/12/028

King, W.P., Kenny, T.W., Goodson, K.E G.L., Cross, W., Despont, M., Durig, U., Rothuizen, H., Binnig, G., Vettiger, P., 2002," Design of Atomic Force Microscope Cantilevers for Combined Thermomechanical Writing and Thermal Reading in Array Operation, 2002," J. Microelectromech. Syst. 11, 765-774. doi:10.1109/JMEMS.2002.803283

King, W.P., Kenny, T.W., Goodson, K.E., Cross, G., Despont, M., Durig, U., Rothuizen, H., Binnig, G.K., Vettiger, P., 2001," Atomic Force Microscope Cantilevers for Combined Thermo-Mechanical Data Writing and Reading," Appl. Phys. Lett. 78, 1300-1302. doi:10.1063/1.1351846

Kohno, H, Bathe, K.J., 2005 “A Flow-Condition-Based Interpolation Finite Element Procedure for Triangular Grids," Int J Numer Methods Fluids 49, 849-75.

doi:10.1002/fld.1027

Kohno, H, Bathe, K.J., 2005, "Insight into the Flow-Condition-Based Interpolation Finite Element Approach: Solution of Steady-State Advection-Diffusion Problems," Int J Numer Methods Eng 63, 197217.

doi:10.1002/nme.1276

Kohno, H, Bathe, K.J., 2005, "Insight into the Flow-Condition-Based Interpolation Finite Element Approach: Solution of Steady-State Advection-Diffusion Problems," International Journal for Numerical Methods in Engineering 63, 197-217.

doi:10.1002/nme.1276

Kohno, H, Bathe, K.J., 2006, "A Nine-Node Quadrilateral FCBI Element for Incompressible Fluid Flows," Commun Numer Methods Eng 22, 917-31.

doi: $10.1002 / \mathrm{cnm} .861$
Kohno, H, Bathe, K.J., 2006, "A Nine-Node Quadrilateral FCBI Element for Incompressible Fluid Flows," Commun Numer Methods Eng 22, 917-31.

doi:10.1002/cnm.861

Lai, J., Perazzo, T., Shi, Z., Majumdar, A.,1997, " Optimization and Performance of High-Resolution Micro-Optomechanical Thermal Sensors," Sens. Actuat. A: Phys. 58, 113-119. doi:10.1016/S09244247(96)01401-X

Lee, J., King, W.P., 2007," Microcantilever Actuation via Periodic Internal Heating," Review Scientific Instruments 78, 054901-6. doi:10.1063/1.2818805

Lee, J., King, W.P., 2008," Microthermogravimetry using a Microcantilever Hot Plate with Integrated Temperature-Compensated Piezoresistive Strain Sensors," J. Appl. Phys. 79, 1-6. doi:10.1063/1.2913337

Lim, K-M, Li, H., 2007, “A Coupled Boundary Element/Finite Difference Method for Fluid-Structure Interaction with Application to Dynamic Analysis of Outer Hair Cells," Comput Struct 85, 911-922. doi:10.1016/j.compstruc.2007.01.003

Mahjoob, S., Vafai, K., and Beer, N. R., 2008, "Rapid Microfluidic Thermal Cycler for Polymerase Chain Reaction Nucleic Acid Amplification" International Journal of Heat and Mass Transfer, 51, 2109-2122.

doi:10.1016/j.ijheatmasstransfer.2007.11.014

Masters, N., Ye, W., King, W.P., 2005,” The Impact of Sub-Continuum Gas Conduction on the Sensitivity of Heated Atomic Force Microscope Cantilevers," Phys. Fluids 17, 100615-8. doi:10.1063/1.1932313

Mosbach, K., Danielsson, B., 1974," Enzyme Thermistor, Biochim. Biophys. Acta 364, 140-145.

Nelson, B.A., King, W.P., Laracuente, A., Sheehan, P.E., Whitman, L.J., 2006," Direct Deposition of Continuous Metal Nanostructures by Thermal Dip-Pen Nanolithography," Appl. Phys. Lett. 13, 033104-3. doi:10.1063/1.2164394

Park, K., Lee, J., Bhargava, R., King, W.P., 2008,” Routine FemtogramLevel Chemical Analyses using Vibrational Spectroscopy and SelfCleaning Scanning Probe Microscopy Tips," Anal. Chem. 80, 32213228.

doi:10.1021/ac702423c

Pinnaduwage, L.A., Gehl, A., Hedden D.L., Muralidharan, G., Thundat, T., Lareau, R.T., Sulchek, T., Manning, L., Rogers, B., Jones, M., Adams, J.D., 2003," A Microsensor for Trinitrotoluene Vapour," Nature 425, $474-474$.

doi: $10.1038 / 425474$ a

Ramos, D, Mertens, J, Calleja, M, and Tamayo, J, 2007," Study of the Origin of Bending Induced by Bimetallic Effect on Microcantilever," Sensors, 7, 1757-1765. doi:10.3390/s7091757

Rinaldi, G, Packirisamy, M, and Stiharu, I, 2007, "Quantitative Boundary Support Characterization for Cantilever MEMS," Sensors 7, 2062-2079.

doi: $10.3390 / \mathrm{s} 7102062$

Sheehan, P.E., Whitman, L.J., King, W.P., Nelson, B.A., 2004," Nanoscale Deposition of Solid Inks via Thermal Dip Pen Nanolithography," Appl. Phys. Lett. 85, 1589-1591. doi:10.1063/1.1785860

Subramanian, A., Oden, P.I., Kennel, S.J., Jacobson, K.B., Warmack, R.J., Thundat, T., Doktycz, M.J., 2002, "Glucose Biosensing using an Enzyme-Coated Microcantilever,” Appl. Phys. Lett. 81, 385-387. 


\section{$\underline{\text { doi: } 10.1063 / 1.1492308}$}

Thundat, T., Sharp, S.L., Fisher, W.G., Warmack, R.J., Wachter, E.A., 1995, "Micromechanical Radiation Dosimeter," Appl. Phys. Lett. 66, $1563-1565$.

doi: $10.1063 / 1.113647$

Thundat, T., Wachter, E.A, Sharp, S.L., Warmack, R.J., 1995,’Detection of Mercury-Vapor Using Resonating Microcantilevers, "Appl. Phys. Lett., 66, 1695-1697. doi:10.1063/1.113896

Thundat, T., Warmack, R.J., Chen, G.Y., Allison, D.P., 1994, “Thermal and Ambient-Induced Deflections of Scanning Force Microscope Cantilevers," Appl. Phys. Lett. 64, 2894-2896. doi:10.1063/1.111407

Thundat, T., Warmack, R.J., Chen, G.Y., Allison, D.P., 1994,” Thermal and Ambient-Induced Deflections of Scanning Force Microscope Cantilevers," Appl. Phys. Lett. 64, 2894-2896. doi:10.1063/1.111407

Tijsseling, A.S., 2007, "Water Hammer with Fluid-Structure Interaction in Thick-Walled Pipes," Comput Struct 85, 844-851. doi:10.1016/j.compstruc.2007.01.008

Vafai, K, and Khaled, A.R.A., 2010, "Innovative Biosensors for Chemical and Biological Assays”, US Patent \# 7,695,951.

Vafai, K., and Khaled, A.R.A., 2010, "Methods and Devices Comprising Flexible Seals, Flexible Microchannels, or Both for Modulating or Controlling Flow and Heat", US patent to be issued in 2010 .

Vafai, K., and Khaled, A.R.A., 2010, "Cooling Enhancements and Control of Flow and Thermal Conditions in Thin Films Supported by Flexible Complex Seals: Applications to Electronic Cooling, Biotechnology and Internal Combustion", US patent \#7, 654,468.
Vafai, K., Ozkan, R., Haddon, R., Khaled, A.R.A., and Yang, M., 2007, "Microcantilevers for Biological and Chemical Assays and Methods of Making and Using Thereof", US Patent \# 7, 288, 404.

Vettiger, P., Cross, G., Despont, M., Drechsler, U., Duerig, U., Gotsmann, B., Haberle, W., Lantz, M., Rothuizen, H., Stutz, R., Binnig, G., 2002," The Millipedenano Technology Entering Data Storage," IEEE Trans. Nanotechn. 1 , 39-64. doi:10.1109/TNANO.2002.1005425

Wu, G.H., Datar, R.H., Hansen, K.M., Thundat, T., Cote, R.J., Majumdar, A., 2001, "Bioassay of Prostate-Specific Antigen (PSA) using Microcantilevers," Nature Biotechnol. 19, 856-860. doi:10.1038/nbt0901-856

Wu, MC, Chang, JS, Wu, KC, Lin, CH, and Wu, CY, 2007," The Effect of Flow Velocity on Microcantilever-Based Biosensors," J. Mechanics 23, 353-358.

Yang, M., Zhang, X., Vafai, K., and Ozkan, C., 2003, "High Sensitivity Piezoresistive Cantilever Design and Optimization for AnalyteReceptor Binding,“ Journal of Micromechanics and Microengineering 13, 864-872. doi:10.1088/0960-1317/13/6/309

Zhang, H, Zhang, X.L, Ji, S.H, Guo, Y.H, Ledezma, G, Elabbasi, N, et al., 2003, "Recent Development of Fluid-Structure Interaction Capabilities in the ADINA System," Comput Struct 81, 1071-85. doi:10.1016/S0045-7949(03)00009-9

Zhang, X., Yang, M., Vafai, K., and Ozkan, C., 2003, "Design and Analysis of Microcantilevers for Biosensing Applications, “ Journal of Association for Laboratory Automation 8, 90-93. doi:10.1016/S1535-5535(04)00264-3 